\title{
LA GESTIÓN DE LA CALIDAD INSTITUCIONAL EN LA UNIVERSIDAD NACIONAL, COSTA RICA
}

\author{
Rónald Miranda Chavarría*
}

Basado en la experiencia lograda en la Universidad Nacional en materia de gestión de la calidad institucional entre los años 2000 y 2004 y con el apoyo de la cooperación holandesa, se plantea la conveniencia de construir, con rigor académico, un concepto de gestión de la calidad amplio e integral, que involucre lo académico, los servicios de apoyo y la gestión. La adopción de un enfoque que conjuga lo estratégico con procesos constructivistas y participativos ha posibilitado edificar una cultura y una visión de futuro de calidad. La experiencia de mejora da cuenta de algunos logros concretos, tales como la desconcentración en la toma de decisiones, la autoevaluación con fines de mejora o de acreditación de las carreras y la conformación de equipos de mejora en los servicios administrativos. Se reconoce que al asumir una gestión de calidad institucional, se establece una brecha de gestión entre las prácticas de gestión imperantes y el modelo propuesto; cerrar esa brecha exige nuevos roles por parte de las autoridades académicas, las cuales deben comprender que la gestión universitaria requiere de un alto rigor y profesionalismo para ser de calidad.
Proposal on the convenience of constructing a broad, integrated concept of quality management that includes academic aspects, support services and the management itself. It is set forth based on the experience achieved at Universidad Nacional on institutional quality management from 2000 to 2004; it has been supported by Dutch cooperation programs. Adopting an approach that fits together strate gic aspects with constructivist and participative processes has afforded the construction of a culture and a vision of a future of quality. Some of the improvements account for achievements such as the diversification of decision making, self evaluation for self improvement or for accreditation purposes of study programs, and the establishment of improvement teams for management services. It is also acknowledged that by adopting an institutional quality management position, a gap is created between the current management procedures and those presented in the proposed model. Closing this gap will require academic authorities to play new roles and to understand that in order to build high-quality university management, it is necessary to work with a lot of precision and professionalism.

* Máster en Administración de Empresas y de Recursos Naturales. Director Programa de Gestión de la Calidad Institucional. Vicerrectoría Académica. Universidad Nacional. Heredia, Costa Rica. 


\section{Introducción}

La gestión de la calidad en el contexto de las instituciones universitarias es un tema que ha cobrado un gran interés y dinamismo, especialmente a partir de la última década del siglo XX. Aunque se dispone de modelos de gestión de la calidad validados en diversos contextos empresariales (Korte, 2002;) o aunque se han desarrollado sistemas particulares para asegurar la calidad académica, tales como los sistemas de evaluación y acreditación de carreras (Díaz-Barriga, 2002; Lepeley, 2001), el tema de la gestión de la calidad en la práctica de las instituciones universitarias plantea desafíos que generan una gran necesidad de aprendizaje y de intercambio de experiencias. Este artículo recoge los fundamentos de la experiencia generada en la Universidad Nacional, con sede en Heredia, Costa Rica, en materia de gestión de la calidad institucional en el período 2000-2004. La experiencia muestra la conveniencia de construir un concepto de gestión de la calidad amplio, acorde con la realidad de la universidad en particular, pero con el debido rigor académico; además, revela la conveniencia de adecuar los procesos metodológicos a la dinámica y la cultura organizativa, con una clara visión de la necesidad de cambiar los modelos y las prácticas de gestión y de trabajo, tanto en lo académico como en los servicios de apoyo.

\section{Antecedentes}

La aspiración por la calidad en la Universidad Nacional surge desde su fundación. Benjamín Núñez, el Rector fundador, concebía una universidad de excelencia. Desde sus orígenes, la institución fue posicionada como la Universidad Necesaria, lo cual se sustentaba en dos importantes dimensiones: la vocación social o histórica y la excelencia académica. No obstante, el esfuerzo por hacer realidad el primer aspecto, fue más contundente que la búsqueda de la materialización de la excelencia académica. La vocación histórica fue descrita por Benjamín Núñez (1974, p. 19) en términos de la "responsabilidad de contribuir al estudio y a la solución de los problemas de crecimiento productivo del país, de integración solidaria de su población,... de preservación y perfeccionamiento de la democracia en Costa Rica". La Universidad fue capaz de desarrollar políticas y sistemas operativos congruentes que permitieron poner en práctica ese concepto. Por ejemplo, la orientación conceptual, práctica y vivencial de muchas de las carreras hicieron posible formar profesionales con una capacidad para contribuir solidariamente a la mejora social, cultural, económica y ambiental del país. Además, la extensión universitaria engendró un conjunto de habilidades que materializaron el concepto de universidad necesaria en el ámbito de muchas comunidades y organizaciones sociales concretas. 
Por su parte, la idea de una universidad de excelencia, fue un asunto que formó parte del discurso oficial. En las palabras de Benjamín Núñez:

“¡Excelencia en los programas y métodos de enseñanza! ... ¡Excelencia en el saber!... ¡Excelencia en la investigación!... ¡Excelencia en la extensión!... ¡Excelencia en las relaciones humanas dentro de la comunidad universitaria!..." (Núñez, p. 56).

Aunque se introdujeron innovaciones, como los currículos diseñados con un sistema de certificados constituidos por conjuntos integrados de materias. No es posible hablar de un concepto ni de sistemas que se hayan mantenido sostenidamente para asegurar la excelencia académica de la Universidad Nacional. No fue sino hasta entrados los años noventas en que irrumpen los esfuerzos progresivos y sistemáticos por elevar y asegurar mayores niveles de calidad en el quehacer universitario. Un primer elemento fue la adopción de un lema invitador e inspirador para la comunidad universitaria: "Universidad Nacional: una realidad de excelencia"; sin embargo, la mejora de los procesos internos era incipiente y se generaba sin una estrategia metodológica estructurada.

A partir de 1994, con la cooperación entre la Universidad Nacional en Costa Rica y la Leiden Hogeschool de Holanda se inició un proyecto de aseguramiento de la calidad. En una primera fase se introdujo el tema de la calidad de la oferta docente como un proyecto piloto en dos unidades académicas. Posteriormente, se trascendió al ámbito institucional en el marco de la denominada Reforma Académica (Ficha 1) que pretendía realimentar y mejorar la oferta de la docencia, la investigación y la extensión.

El principal objetivo consistió en desarrollar y poner en ejecución un sistema de mejoramiento de la calidad y una cultura de evaluación. Efectivamente, se desarrolló un sistema de evaluación denominado EVA-UNA que utilizó instrumentos especialmente diseñados para examinar todos los programas académicos de la institución. Este estudio involucró cerca de ciento cincuenta académicos, se evaluaron distintas escuelas e institutos en calidad de pares; además, los resultados de las autoevaluaciones institucionales fueron sometidos al análisis crítico de evaluadores externos procedentes de otras instituciones académicas nacionales e internacionales (Mora, 1998).

Como resultado, se actualizó el currículo en la mayoría de las carreras de la universidad, se modificó el calendario académico, se integraron muchos proyectos de investigación y extensión, así como se mejoró la eficacia y la pertinencia de dichos proyectos. Además de estos resultados, el proceso dejó una 
lección clara y desmitificadora acerca de la importancia y las ventajas de un proceso de evaluación. Como consecuencia, se ha desarrollado progresivamente una mayor conciencia de que la calidad es parte de la responsabilidad social de la institución, como universidad pública debemos hacer lo mejor con los recursos que la sociedad invierte en la institución.

\section{Tích 1}

\section{Un hito relevante}

La Reforma Académica constituye el más importante punto de inflexión a nivel institucional que hace girar el rumbo de la universidad hacia mayores niveles de calidad. El Consejo Universitario lo colocó como la acción prioritaria de la Universidad (Mora, 1998).

La reforma académica fue formalmente definida como "el proceso integral mediante el cual la Universidad evalúa, actualiza y reorganiza su quehacer académico, a la luz de su misión histórica, las tendencias del desarrollo nacional e internacional, los avances disciplinarios, los requerimientos estudiantiles y las fortalezas institucionales, buscando elevar la calidad, la magnitud y el impacto de sus acciones en la sociedad" (SCU-721-96).

La reforma académica, con todo lo controversial que pueda resultar para muchos universitarios, constituye, a nuestro modo de ver, el hito más importante que se ha dado en la Universidad en materia de evaluación y mejoramiento de la calidad académica.

\section{El Programa de Gestión de la Calidad Institucional}

Uno de los aprendizajes del proceso de reforma académica consistió en reconocer que aun cuando se evalúen y rediseñen de la mejor manera los procesos académicos, dichos esfuerzos de mejora son insuficientes si no se realizan mejoras en la gestión institucional y en los servicios administrativos. Ese fue el fundamento para desarrollar un importante proyecto institucional que hoy se denomina Programa de Gestión de la Calidad Institucional. El objetivo inicial consistió en el "fortalecimiento de la capacidad de poner en práctica un Sistema de Gestión de la Calidad integral, efectivo y participativo para la ampliación de la oferta académica, los servicios administrativos y la planificación y la gestión" de la Universidad Nacional.

Esto con la meta de alcanzar una calidad completa en la institución. 


\section{Enfoque de la Gestión de la Calidad Institucional}

El trabajo realizado durante el cuatrenio 2000-2004 ha sido el más intensivo que se ha realizado en la institución en el campo de la calidad. En atención a sus objetivos, el programa realiza actividades en cuatro ámbitos temáticos: la calidad académica como propósito último de mejoramiento, en función del cual están todos los demás esfuerzos de mejora; la calidad en la planificación con miras a orientar estratégicamente el desarrollo futuro de la institución; la calidad en la gestión institucional y la calidad en los servicios de apoyo a la academia. Aparte de esos ámbitos, la comprensión de la naturaleza de la organización universitaria ha constituido un importante fundamento en la construcción del enfoque, del concepto y del manejo metodológico de los procesos de gestión de la calidad institucional.

\section{La comprensión de la naturaleza de la organización universitaria}

Las universidades consisten en comunidades de académicos y estudiantes que cuentan con el apoyo de funcionarios administrativos para cumplir su misión de desarrollar el conocimiento. Por tanto, deben entenderse fundamentalmente como organizaciones de profesionales académicos (Mintzberg, 1983). Las decisiones son tomadas principalmente por los académicos que son profesionales formados en las más diversas disciplinas. Ellos constituyen la garantía del buen funcionamiento de la organización, pero asumen patrones de trabajo que demandan autonomía, libre pensamiento y mínima supervisión directa (Baldridge, 1983). En la base de la pirámide organizativa se encuentran personas con una alta capacidad crítica e intelectual, lo cual lleva a comportamientos organizativos difícilmente modelables por la gestión superior.

Los académicos necesariamente irrumpen en las funciones de la administración, ya sea en función de los proyectos académicos, o en la conducción formal en los cargos del gobierno universitario o en la dirección académica de las facultades, vicerrectorías u otros cuerpos colegiados. En tales casos las habilidades en juego usualmente no proceden de una sólida formación en gestión sino del talento natural, de la intuición, del autodidactismo o incluso por efecto de la consejería mutua entre académicos experimentados o de expertos administrativos encargados de las funciones de apoyo. Este rasgo de bajo profesionalismo en la gestión, introduce complejidades en la cultura organizativa tales como la ambigüedad de objetivos (Baldridge, 1983), la anarquía organizada de los académicos (Ellström, 1983; Baldridge, 1983) y los conflictos burocráticos (Bacharach et al, 1991). 
En las universidades se dan otras complejidades estructurales. Por ejemplo, comúnmente se generan numerosas estructuras o aparatos de apoyo administrativo, muchas veces inspiradas en la organización del estado, lo cual las convierte en entidades altamente burocráticas (Mintzberg, 1983). El paradigma de la república universitaria, surgido en la Reforma de Córdoba, subyace en la estructura de muchas universidades públicas latinoamericanas (Universidad de Antioquia, 1997; España, 1998); es un concepto que lleva a la preeminencia de estructuras formales altamente complejas, diversificadas y rígidas en las cuales la gestión se torna altamente politizada y encuentra grandes dificultades para estandarizar los procedimientos administrativos y para propiciar la innovación y la flexibilidad ${ }^{1}$.

A pesar de su naturaleza compleja, las universidades pueden alcanzar patrones de funcionamiento aceptables en tanto se encuentren en entornos estables (Mintzberg, 1983), pero en la medida en que el entorno se vuelve inestable y la actividad académica genera mayores innovaciones, se manifiestan las disfuncionalidades estructurales, las cuales son síntomas de la necesidad de una nueva concepción de la gestión universitaria.

\section{El concepto de calidad institucional}

Al comprender la naturaleza y la cultura de la organización, estamos en condiciones de explicitar nuestra hipótesis de trabajo: la calidad en la Universidad Nacional se construye a partir de las múltiples nociones y de esfuerzos afines que procuran mejorar el quehacer universitario; es decir, compromete la especificación de la calidad en la actividad académica (en la docencia, la investigación, la extensión y en la producción artística y literaria); la calidad en los servicios de apoyo a la academia en función de la estrategia institucional y la calidad en la gestión y el gobierno universitarios.

El concepto de calidad institucional que se ha venido construyendo y posicionando reconoce la preocupación por mejorar en forma continua en varios elementos cuyo grado de complejidad es creciente, La calidad básica, es decir, el deseo de las personas de servir y mejorar continuamente; la calidad de los productos del quehacer institucional; la calidad en los procesos fundamentales de la academia, la docencia, la investigación, la extensión y la producción.

1 "Una declaración más explícita acerca del cambio en el concepto de universidad se ha dado en la Universidad de Antioquia. El nuevo concepto parte de considerar a la institución universitaria como un patrimonio de la sociedad y que su función social central es la academia. Este postulado impacta la organización, específicamente en la configuración de los órganos colegiados superiores de conducción ya que se procura una representatividad de la sociedad. Este concepto de universidad supera la concepción de universidad como una especie de república, bajo la cual, la gestión académica queda subordinada a una función política que pasa a ser el centro de la vida universitaria" (Miranda, et al, 2001). 
artística y académica; la efectividad y la flexibilidad en los sistemas, las normas y procesos administrativos; la calidad en la gestión institucional: las estructuras de gobierno, los sistemas de toma de decisión y el perfil de los gestores; la calidad en cuanto a la pertinencia, es decir, el valor y significado para la sociedad del quehacer institucional.

En síntesis, una institución de calidad cuenta, de manera sostenida, con una estructura efectiva para alcanzar la misión y la visión propuestas, con sistemas eficaces y ransparentes en la toma de decisiones, con altas competencias y confiabilidades en los gestores y con personas cuyos talentos, valores y acciones se potencian para servir y alcanzar la misión institucional, tanto en su dimensión académica como social.

\section{Un proceso con múltiples esfuerzos}

Particularmente en la Universidad Nacional, donde la noción de constructivismo está fuertemente enraizada, no resulta acertada la implantación de modelos de gestión de la calidad, por más probados que estén en otros contextos. Sin embargo, es posible lograr consensos progresivos acerca de cuál pudiera ser la mejor estrategia para gestionar la calidad.

Estas nociones y esfuerzos se ven impulsados por la existencia de políticas institucionales o de programas o proyectos como el de Gestión de la Calidad Institucional, el de Evaluación Académica, Desarrollo Curricular, Gestión de Proyectos, Gestión del Servicio y Desarrollo Humano, Auditoría de la Calidad y la Oficina de Cooperación Internacional, entre otros. Sin embargo, hay muchos esfuerzos de calidad implícita que se manifiestan en mejoras desarrolladas por iniciativa de los académicos o de funcionarios administrativos que en un esfuerzo diario, actúan impulsados por el deseo, el compromiso, la curiosidad, la creatividad, el ingenio y hasta por un cierto aventurerismo. Esto significa que la calidad se ha venido gestionando tanto de manera formal como informal y se promueve con el esfuerzo de numerosas instancias, lo cual demuestra el arraigo de una vocación por la calidad en la vida universitaria; además, revela la existencia de las capacidades básicas para gestionar la calidad.

Dentro de ese panorama, los esfuerzos del Programa de Gestión de la Calidad se han volcado a elevar capacidades, construir conocimientos, desarrollar habilidades y alimentar las voluntades o deseos por hacer mejoras cualitativas. Específicamente, se ha hecho una importante inversión en capacitación de funcionarios sobre temas relacionados con la calidad en el servicio, autoevaluación, acreditación, planificación y desarrollo humano. A lo largo de numerosos talleres de trabajo se ha fortalecido a las autoridades de distintos niveles mediante el 
desarrollo de nuevas habilidades y herramientas de gestión. La construcción de una visión prospectiva de la universidad se ha hecho de manera participativa, conjugando las perspectivas de la docencia, la estudiantil, de la investigación, la extensión y la gestión. Otras acciones se han orientado a construir y reforzar vínculos con otros procesos institucionales para alinear estratégicamente distintos procesos y esfuerzos de mejora. Ha resultado muy estimulante promover el intercambio y el trabajo conjunto por parte de distintos actores internos interesados y motivados por la calidad, puesto que estas acciones orientan el clima institucional hacia la calidad: la intención es estimular una serie de liderazgos laterales para promover la calidad y el cambio a lo largo y ancho de la institución. En la Universidad Nacional, muchos de estos liderazgos se ubican en programas de apoyo a la gestión y tienen un alto poder de influencia tanto en los niveles de autoridad como en el trabajo académico y administrativo de las facultades. El apoyo a órganos e instancias de conducción superior en el análisis y la formulación de nuevas políticas sobre temas estratégicos para la institución ha permitido ampliar la voluntad y el compromiso de las distintas autoridades con los procesos de mejora y de cambio institucional y especialmente está llevando a una toma de decisiones concordantes con la nueva concepción estratégica y de calidad que se viene construyendo.

\section{El papel de la cooperación internacional}

El avance logrado en la Universidad Nacional en materia de gestión de la calidad institucional ha sido facilitado en gran medida por la cooperación brindada por varias universidades holandesas. Hogeschool Leiden fue la institución que tomó la iniciativa conjuntamente con la Universidad Nacional para impulsar la gestión de la calidad. La Universidad Libre de Ámsterdam en cooperación con la Pontificia Universidad Javeriana de Colombia propusieron un enfoque integral y moderno sobre la gestión en el ámbito institucional. La Universidad de Utrecht contribuyó en los procesos de mejora de la calidad en los proyectos de investigación y la Universidad de Tilburg, en el tema de la gestión de la información y la tecnología.

Sobre la base de compartir experiencias, la cooperación internacional ha estimulado procesos en los cuales la Universidad Nacional identifica sus propios desafíos y adopta sus propias decisiones acerca de cómo gestionar la calidad en la institución. El seguimiento y acompañamiento permitieron realimentar las decisiones de manera coherente. La cooperación internacional también posibilitó indagar acerca de los sistemas de gestión directamente en universidades de Holanda, Colombia y Chile, así como ampliar vínculos de información con 
universidades de varios otros países de Iberoamérica. Estas interacciones permiten un conocimiento actualizado de las prácticas y los conceptos de gestión que se están utilizando en las universidades en el ámbito internacional.

\section{Avances relevantes}

\section{Un nuevo concepto de gestión}

El nuevo concepto de gestión institucional adopta la calidad como idea rectora de la gestión universitaria. En función de esa idea, se dispone de tres medios fundamentales e interdependientes: la gestión estratégica, la desconcentración y la rendición de cuentas. El objeto del nuevo concepto de gestión está dado por cuatro temas relevantes de cambio. El primero es la organización de lo académico, que constituye quizá el mayor desafío dado que imumpe en las tradicionales fronteras de las disciplinas y los compartimentos universitarios; se trata de un esfuerzo de largo plazo que requiere de criterios flexibles, orientados por los principios de potenciación de los conocimientos universitarios y la búsqueda de la sinergia en el empleo de los talentos de que dispone la institución. El segundo es la estructura institucional; referida a establecer tres niveles de gestión con una desconcentración hacia las facultades de muchos de los procesos y servicios centrales. Este tema comporta replantear las principales funciones y relaciones que se establecen entre los distintos niveles de gestión institucional y el nuevo papel y organización que deben asumir los servicios de apoyo cuando se desconcentran en las facultades. El tercer tema de cambio se refiere a la gestión integral del talento humano, el cual consiste en dar una función más estratégica a la hasta entonces funcional y burocráticamente entendida administración de recursos humanos. Finalmente, está el tema de la gestión de la información y la tecnología como herramienta para la oportuna toma de decisiones y para potenciar la producción académica institucional.

\section{El proceso de desconcentración}

La puesta en práctica de dicho concepto debe verse como un proceso gradual de largo plazo. Un punto de partida ha consistido en la realización de un proyecto piloto de desconcentración de un tema clave que durante los últimos años ha constituido una decisión centralizada en la Rectoría. En este caso se decidió desconcentrar las decisiones relacionadas con el uso de los recursos liberados por funcionarios que se jubilan, renuncian o fallecen. Estas decisiones pasan a tomarse en las facultades, donde un decano con su Consejo Académico 
de facultad, serán los responsables de decidir en qué se utilizarán los recursos que se liberan en el ámbito de la facultad. El propósito de la experiencia piloto es establecer aprendizajes sobre las prácticas y las herramientas requeridas en un modelo de gestión desconcentrada y con rendición de cuentas.

La toma de decisiones en forma desconcentrada responde al concepto de que la calidad de la gestión depende de la calidad de las decisiones. Por tanto, se ha hecho un esfuerzo en la preparación de las autoridades de las facultades (decanos y Consejos Académicos) para la comprensión de la nueva dinámica de toma de decisiones. Esto incluye la necesidad de comprender tres herramientas básicas para la desconcentración: los acuerdos de gestión, los sistemas de asignación de recursos y la rendición de cuentas (Fichas 2, 3 y 4).

\section{Trotur 2}

\section{Los acuerdos de gestión}

Son acuerdos entre dos autoridades de diferentes niveles. Se basan en el Plan Global Institucional, los planes de desarrollo de las facultades y las unidades académicas que conforman una facultad.

Los acuerdos pueden contemplar los siguientes aspectos:

- Responsabilidades conferidas por parte de la autoridad superior.

- Situación inicial o punto de partida respecto al tema desconcentrado.

- Resultados esperados y sus plazos.

- Recursos asignados.

- Parámetros o referentes de calidad o cantidad.

- Forma y momento para el seguimiento (rendición de cuentas).

- Consecuencias. 


\section{Ficha 3}

\section{Los criterios de asignación de recursos}

La asignación de recursos debe estar orientada hacia objetivos estratégicos que deben expresarse en criterios específicos de asignación. Estos deben superar la asignación histórica del presupuesto. Dos métodos de asignación de recursos que se podrían utilizar son los siguientes:

\section{La elaboración de un algoritmo}

Se trata de un modelo matemático que especifica los criterios establecidos y las tasas de asignación de recursos, las cuales varían en función de la calificación otorgada a cada criterio. Este método requiere de la validación de los factores determinantes de los recursos y la calibración de las tasas de asignación.

Ejemplo: los factores y las tasas del ejemplo se presentan a modo de ilustración.

Presupuesto laboral asignado a una carrera

\section{Determinantes de recursos \\ Tasa de asignación}

$\mathrm{N}^{\circ}$ estudiantes matriculados

2,0 jornadas académicas por cada 100 estudiantes.

$\mathrm{N}^{\circ}$ horas laboratorio

0,25 jornadas académicas por cada grupo de laboratorio.

$\mathrm{N}^{\circ}$ de graduados el año anterior

0,50 jornadas académicas por cada 100 graduados.

\section{Mediante criterios de referencia}

No se trata de tener criterios claves que sean funcionales y que sean conocidos de antemano tanto por quienes toman las decisiones como por los usuarios de los recursos.

En este caso los criterios deben estar correlacionados con factores de absorción de recursos; por ejemplo, el "número de académicos jubilados durante el último año" se relaciona mejor con los recursos necesarios que un criterio como "importancia para el país de la carrera".

Los recursos se asignan tomando en consideración dichos criterios en forma global, pero no necesariamente se establecen tasas de asignación vis a vis. De manera que es posible considerar aspectos cualitativos relacionados con el desempeño, la productividad académica, las necesidades, los proyectos futuros o cualquier situación especial que se presente. 


\section{La rendición de cuentas}

En función del tema o el tipo de decisión que se desconcentra, se elabora un pequeño protocolo para la rendición de cuentas. En él se establece lo siguiente:

1. Quién rinde cuentas ante quién.

2. Acerca de qué se rinde cuentas:

- El ejercicio de las responsabilidadesconferidasen los acuerdos de gestión.

- Los logros alcanzados o los avances de acuerdo con los indicadores de cumplimiento convenidos.

- El uso de los recursos que han sido confiados.

- Los problemas surgidos y las medidas adoptadas en el proceso de gestión.

3. La periodicidad de las reuniones de rendición de cuentas.

4. Las consecuencias por el buen o mal desempeño.

5. Acciones por seguir o adecuación de acuerdos.

Los actos de rendición de cuentas, en este contexto, consisten de momentos de encuentro, de diálogo entre autoridades de dos niveles de gestión directamente relacionados. No es un envío de informes, aunque sí se presentan datos que revelan hechos, pero el énfasis debe estar en el contenido: en las responsabilidades y los objetivos en función de los cuales están los recursos, que sin lugar a dudas deben corresponder con el desarrollo académico y el cumplimiento de la misión institucional.

Los primeros efectos de la experiencia piloto de desconcentración reportados por decanos y miembros de Consejos Académicos de las facultades en talleres de trabajo, revelan que aunque la experiencia es de magnitud limitada, la toma de decisiones se logra en un menor plazo que cuando las decisiones se toman centralizadamente. Asimismo, permite conocer la situación real en términos de necesidades y disponibilidad de recursos de cada una de las unidades académicas que conforman la facultad. Al contar con la información, se tienen mayores elementos a mano para tomar las decisiones lo que a la vez genera transparencia en los Consejos Académicos de las facultades. Se reconoce que la nueva forma de tomar decisiones genera un mayor conocimiento de la facultad 


\section{Los académicos}

necesariamente irrumpen en las funciones de la administración, ya sea en función de los proyectos académicos, o en la conducción formal en los cargos del gobierno universitario o en la dirección académica de las facultades, vicerrectorías u otros cuerpos colegiados. En tales casos las habilidades en juego usualmente no proceden de una sólida formación en gestión sino del talento natural, de la intuición, del

autodidactismo o incluso por efecto de la consejería mutua entre académicos

experimentados o de expertos administrativos encargados de las funciones de apoyo. y propicia la necesidad y el interés mutuo por parte del decanato y las unidades académicas de conocer mejorlos planes estratégicos de unos y otros. La experiencia también revela la necesidad de lograr una mejor comprensión y desempeño del nuevo rol de los servicios de apoyo a las facultades cuando los procesos se desconcentran; por ejemplo, los programas de servicios financieros y de recursos humanos deben responder a resoluciones que comunican los decanos y que tradicionalmente se emanaban desde la Rectoría o la Vicerrectoría respectiva.

\section{Planificación estratégica}

Los avances más concretos y significativos han sido la construcción de una visión compartida sobre el futuro de la Universidad, la introducción de un concepto de estrategia universitaria basada en habilidades, para ordenar metodológicamente un proceso participativo de elaboración

del Plan Global Institucional de mediano plazo.

Este proceso ha revelado que el desarrollo futuro de la Universidad estará muy determinado por la capacidad que adquieran las autoridades académicas y administrativas para hacer del Plan Global el principal referente para la toma de decisiones. Por eso este contempla varios factores clave para su puesta en ejecución, entre los cuales se destacan dos: el primero se trata del desarrollo de un programa de capacitación en gestión universitaria que eleve el perfil de competencias y confiabilidades de las autoridades universitarias en los diferentes niveles de gestión. Esto aportaría a las autoridades académicas los conocimientos, las habilidades y las herramientas de gestión requeridas para actuar en concordancia con el nuevo concepto de gestión institucional y para ser capaces de ejecutar la estrategia institucional contemplada en el Plan Global. El segundo 
factor clave consiste en la conveniencia de introducir y hacer uso de una herramienta de gestión estratégica: se trata de la introducción del sistema de conducción integral que mediante un conjunto de indicadores clave, facilita a las autoridades el monitoreo del cumplimiento de los objetivos estratégicos establecidos en el Plan Global².

\section{La calidad académica}

La mejora de la gestión en los procesos relacionados con la oferta docente, se promueve desde la dirección de docencia. La gestión de los programas y los proyectos de investigación, extensión y producción se realiza desde las direcciones de Extensión e Investigación.

El principal proceso de mejora en la calidad de la docencia se realiza mediante procesos de autoevaluación con fines de mejoramiento y de acreditación (Universidad Nacional, 2004). También se realizan procesos de rediseño curricular de varias carreras acompañados de una adecuación de los ciclos lectivos, con lo cual se procura organizar la temporalidad de los procesos de enseñanza y aprendizaje de modo que respondan mejor a las aspiraciones de los estudiantes y los profesores. Una iniciativa reciente se relaciona con el desarrollo de un modelo pedagógico diferenciador, acorde con los principios de la institución y que, entre oros rasgos, responda a los desafíos de la sociedad del conocimiento y de la tecnología.

En la gestión de proyectos, los esfuerzos incipientes se han enfocado a definir una visión de futuro de la investigación y la extensión y a identificar factores clave para un sistema de calidad de la investigación. Los modelos de acreditación de laboratorios constituyen una oportunidad aplicable a los casos en los que se venden servicios en los cuales la confiabilidad de los resultados, por sus alcances legales o comerciales, se requiere del respaldo de los procedimientos y las técnicas utilizadas.

Lo que se ha podido identificar es que la calidad de la gestión de los proyectos tiene una dimensión externa y otra interna. En lo externo compromete a todo el quehacer investigativo a conocer con rigurosidad los intereses y las necesidades de conocimiento e información de diferentes usuarios, científicos, productores, instituciones, comunidades o redes. También es necesario el conocimiento de las políticas de los gobiernos o donantes relacionadas con el desarrollo científico y social.

2 Se trata del Balanced scoredcard, que ha sido traducido como Anotador Balanceado, Cuadro de Mando Integral o Tablero de Comando. Fue diseñada con el propósito de generar indicadores que aseguren la ejecución de una estrategia, pero a partir de su utilización se ha convertido en todo un sistema de gestión (Kaplan). 
En lo interno, se trata de ocuparse tanto de la calidad de la gestión de la investigación como de la calidad en la acción investigativa. Lo primero supone la adecuación de las políticas, los procesos y los incentivos relacionados con la investigación de modo que sean menos burocráticos, más desconcentrados y efectivos; además, el aseguramiento de las condiciones de apoyo como la disponibilidad de información oportuna sobre opciones de cooperación, los flujos de comunicación entre académicos y las instancias administrativas y el acompañamiento de la Vicerrectoría a los procesos de gestión en las facultades.

El mejoramiento de la acción investigativa corresponde a la naturaleza del trabajo que ejecutan los equipos de investigadores; comporta el liderazgo y la organización interna y mutua de los proyectos para potenciar el intercambio, la reflexión académica, el uso de los recursos, los alcances y el impacto de las investigaciones. Es fundamental la atención a las cualidades científicas de la investigación: la rigurosidad científica o académica, la relevancia, la pertinencia y la efectividad de los vínculos con la docencia y los posgrados. Además, la calidad de los resultados de la investigación debe reflejar los niveles de productividad académica así como la utilidad y el ámbito de difusión e impacto de la investigación universitaria.

Finalmente, la gestión de la calidad de los proyectos, tanto de investigación como de extensión, debe someterse a evaluaciones de calidad que como parte de un sistema, debe contener parámetros e instrumentos que permitan dar cuenta de si los niveles de calidad de los proyectos son adecuados a los referentes nacionales o a referentes internacionales de calidad.

\section{La calidad en los servicios de apoyo}

El mejoramiento de la calidad en los servicios se ha introducido a partir de un proceso de capacitación dirigido a funcionarios administrativos de las diferentes vicerrectorías. Inicialmente, las actividades de capacitación introdujeron el referente del modelo de excelencia de la Fundación Europea de Gestión de la Calidad (1999), dada su adaptación metodológica para instituciones de educación superior. Sobre esa base se iniciaron pequeñas experiencias de mejoramiento bajo el concepto que aquí se denominó "calidad básica"3. Los procesos de orientación y atracción estudiantil, de pagos a estudiantes, de atención a estudiantes en la oficina de registro y la capacitación para la formulación de los planes anuales operativos son algunos de los procesos con los que se trabajó bajo esta concepción. iniciativa de mejoramiento. El propósito es crear un sentido de logro e ir introduciendo una cultura de mejoramiento continuo que luego trasciende al mejoramiento de temas más complejos como procesos, sistemas y estructuras. 
Posteriormente, se instituyó dentro del Programa de Recursos Humanos el proyecto de Fortalecimiento de la gestión del servicio y desarrollo humano ${ }^{4}$. Desde ese momento la Vicerrectoría de Desarrollo adquirió un mayor protagonismo en la gestión de la calidad de los servicios. A partir de actividades de capacitación se han establecido cerca de una veintena de grupos de mejora para la evaluación y el rediseño de diversos procesos administrativos.

\section{Aprendizajes relevantes}

La gestión de la calidad institucional en el contexto universitario, enfrenta en la práctica dificultades relacionadas con la naturaleza de la organización de académicos; por ejemplo, los altos niveles de criticidad hacen que no resulte simple poner en práctica modelos de gestión de la calidad validados en otros contextos organizativos. En el proceso de la Universidad Nacional, ha resultado fundamental comprender lo general y lo particular de la cultura organizativa e inducir la cultura de la calidad a partir de acciones participativas en las cuales se generan consensos acerca del tipo de universidad que deseamos para el futuro; así se ha posibilitado el análisis, la discusión y el replanteamiento de temas fundamentales de manera abierta y fraterna. Sobre esa construcción, ha sido importante, aunque en ningún modo sencillo, estimular la toma de decisiones de manera que permita alcanzar de un modo más directo algunos aspectos prioritarios que revelen la capacidad de lograr mejoras, ya sea en la gestión, en los servicios o en la actividad académica.

Aun cuando se han elaborado un nuevo concepto de gestión y un plan global institucional, se abre una brecha de gestión definida por la diferencia entre las prácticas tradicionales de gestión y la manera como debería gestionarse la universidad en el futuro. Una manifestación de la brecha de gestión se da en el proceso de priorización y decisión, el cual resulta complejo debido, entre otros factores, a que la toma de decisiones está muy determinada por órganos colegiados. Esto sucede en todos los niveles de gestión, en la conducción superior, en las facultades y en las unidades académicas.

Cerrar la brecha de gestión es el reto para las futuras generaciones de autoridades académicas. Ellas tendrán que descubrir su nueva responsabilidad como gestores de cambio. Deben preocuparse por comprender y desarrollar las habilidades correspondientes y adquirir el dominio de las nuevas herramientas de gestión.

Es claro que para ver avances y resultados se requiere mucho esfuerzo, perseverancia y alta capacidad de resiliencia. La opción por la calidad requiere

4 El Consejo Universitario aprobó y declaró de interés institucional el proyecto, en la Sesión Ordinaria del 22 de mayo del 2003, según consta en el artículo tercero, inciso VI, acta 2472. 
superar barreras en la cultura, en paradigmas, en prácticas y hábitos que suelen estar fuertemente arraigados en las personas.

Finalmente, aun cuando hay muchos desafíos pendientes, la calidad de lo avanzado se ha estimulado gracias al papel que ha jugado la cooperación internacional, la cual ha dejado una lección importante: la calidad y la competitividad universitaria depende de nuestra capacidad para cooperar mutuamente.

\section{Conclusión}

La gestión de la calidad en una institución universitaria en la actualidad es una actividad que requiere de rigor académico, se necesitan conocimientos, habilidades y herramientas mucho más complejas por parte de las autoridades académicas. Para que estas puedan desempeñar un papel determinante en el cambio institucional, deben reconocer que las prácticas tradicionalmente usadas en la gestión universitaria, resultan insuficientes para realizar una gestión responsable, efectiva y transparente. Se debe actuar en coherencia con un nuevo concepto de gestión de calidad institucional para que la universidad en su conjunto pueda cumplir su misión de manera efectiva. Es importante reconocer los retos que se imponen y estar conscientes de que se trata de un esfuerzo de muy largo plazo que requiere constancia y persistencia; pero lo más valorable lo constituye la decisión por asumir con seriedad y profesionalismo la gestión de la calidad en la institución. Finalmente, es de reconocer que en la Universidad Nacional, el papel de la cooperación intemacional ha sido relevante para orientar e intensificar la gestión de la calidad.

\section{Referencias}

Bacharach, S. B.; Bamberger, P. y Conley, S. C. (1991). Negotiating the "see-saw" of managerial strategy: a resurrection of the study of professionals in organizational theory. Research in sociology of organizations (8), 217-238.

Baldridge, J. V. (1983). Organization characteristics of colleges and universities. In Baldridge and Deal. The dynamics of organizational change in education. Berkekey: Mc Cutchan Publishing Corporation, 38-59.

Díaz-Barriga, A. (2002). La evaluación en el marco de las políticas hacia la educación superior: Retos y perspectivas. Seminario: Desarrollo de la calidad en universidades de América Latina. México, 2001. Eschborn. GTZ: 24-40.

Ellström, P. (1983). Four faces of educational organizations. Higher Education (Amsterdam) 12 (1983), 231-141. 
España, O. (1998). Filosofía e historia de la Universidad. En España, O. Universidad Nacional, Historia y Utopía. Heredia. Editorial Fundación UNA, pp. 29-52.

European Foundation for Quality Management. 1999. Method for improving the quality of higher education based on the EFQM model. Netherlands: HBO Expert Grup.

Kaplan, R. y Norton, D.P. (2001). Cómo utilizar el cuadro de mando integral. Barcelona: Gestión 2000.

Korte, R. (2002). Gestión de calidad: experiencias de la industria y el sector de servicios para ser aprovechadas en las universidades. Seminario: Desarrollo de la calidad en universidades de América Latina. México, 2001. Eschborn: GTZ: 41-53.

Lepeley. M. E. 2001. Gestión y calidad en educación. Un modelo de evaluación. Santiago: Mc Graw Hill.

Mintzberg. H. (1983). The profesional bureaucracy. In Structures in five. Designing effective organizations. Englewood Cliffs: Prentice-Hall, pp. 189-213.

Mintzberg. H. (1988). La organización profesional. En Mintzberg, H. y QUINN, J. B. 1988. El proceso estratégico. Conceptos, contextos y casos.

Miranda, R.; Marín, O.; Ríos, M.; Mora, G.; Gutiérrez, I. y Hernández, C. (2001). La gestión universitaria en cuatro universidades colombianas. Heredia. Universidad Nacional.

Mora, J. (1998). Veinticinco años construyendo el futuro. En España, O. Universidad Nacional, Historia y Utopía. Heredia. Editorial Fundación UNA, pp. 13-27.

Núñez, B. (1974). Hacia la universidad necesaria. Heredia: Universidad Nacional.

Núñez, B. (1983). Una universidad de excelencia. En España, O. Universidad Nacional, Historia y Utopía. Heredia. Editorial Fundación UNA, pp. 56-57.

Universidad Nacional (2004). Acuerdos Generales del Consejo Universitario. SCU-894-2004. Una Gaceta (11), 7-8.

Universidad de Antioquia. (1997). Estatuto General. Acuerdo Superior N ${ }^{\circ} 1$ del 5 de marzo de 1997. Medellín. 